\title{
In vitro, in silico and in vivo study challenges the impact of bronchial thermoplasty on acute airway smooth muscle mass loss
}

\author{
Igor L. Chernyavsky $\mathbb{1}^{1,10}$, Richard J. Russell ${ }^{2,10}$, Ruth M. Saunders ${ }^{2,10}$, \\ Gavin E. Morris ${ }^{3}$, Rachid Berair ${ }^{2}$, Amisha Singapuri ${ }^{2}$, Latifa Chachi ${ }^{2}$, \\ Adel H. Mansur ${ }^{4}$, Peter H. Howarth ${ }^{5}$, Patrick Dennison ${ }^{5}$, Rekha Chaudhuri ${ }^{6,7}$, \\ Stephen Bicknell ${ }^{6}$, Felicity R.A.J. Rose ${ }^{8}$, Salman Siddiqui ${ }^{2}$, Bindi S. Brook ${ }^{9,11}$ \\ and Christopher E. Brightling ${ }^{2,11}$
}

@ERSpublications

Bronchial thermoplasty treatment for asthma has unexpected possible mechanisms of action http://ow.ly/ZcuE30jsaSa

Cite this article as: Chernyavsky IL, Russell RJ, Saunders RM, et al. In vitro, in silico and in vivo study challenges the impact of bronchial thermoplasty on acute airway smooth muscle mass loss. Eur Respir J 2018; 51: 1701680 [https://doi.org/10.1183/13993003.01680-2017].

ABSTRACT Bronchial thermoplasty is a treatment for asthma. It is currently unclear whether its histopathological impact is sufficiently explained by the proportion of airway wall that is exposed to temperatures necessary to affect cell survival.

Airway smooth muscle and bronchial epithelial cells were exposed to media $\left(37-70^{\circ} \mathrm{C}\right)$ for $10 \mathrm{~s}$ to mimic thermoplasty. In silico we developed a mathematical model of airway heat distribution postthermoplasty. In vivo we determined airway smooth muscle mass and epithelial integrity pre- and postthermoplasty in 14 patients with severe asthma.

In vitro airway smooth muscle and epithelial cell number decreased significantly following the addition of media heated to $\geqslant 65^{\circ} \mathrm{C}$. In silico simulations showed a heterogeneous heat distribution that was amplified in larger airways, with $<10 \%$ of the airway wall heated to $>60^{\circ} \mathrm{C}$ in airways with an inner radius of $\sim 4 \mathrm{~mm}$. In vivo at 6 weeks post-thermoplasty, there was an improvement in asthma control (measured via Asthma Control Questionnaire-6; mean difference 0.7, 95\% CI 0.1-1.3; $\mathrm{p}=0.03$ ), airway smooth muscle mass decreased (absolute median reduction 5\%, interquartile range (IQR) $0-10 ; \mathrm{p}=0.03$ ) and epithelial integrity increased $(14 \%$, IQR 6-29; $\mathrm{p}=0.007)$. Neither of the latter two outcomes was related to improved asthma control.

Integrated in vitro and in silico modelling suggest that the reduction in airway smooth muscle postthermoplasty cannot be fully explained by acute heating, and nor did this reduction confer a greater improvement in asthma control.

This article has supplementary material available from erj.ersjournals.com

Received: Aug 172017 | Accepted after revision: March 292018

Copyright OERS 2018. This ERJ Open article is open access and distributed under the terms of the Creative Commons Attribution Licence 4.0. 
Affiliations: 'School of Mathematics, University of Manchester, Manchester, UK. ${ }^{2}$ Dept of Infection, Immunity and Inflammation, Institute for Lung Health, NIHR Leicester Biomedical Research Centre, University of Leicester, Leicester, UK. ${ }^{3}$ Dept of Cardiovascular Sciences, University of Leicester, Leicester, UK. ${ }^{4}$ Heart of England NHS Trust, Birmingham, UK. ${ }^{5}$ Clinical and Experimental Sciences, University of Southampton, Southampton NIHR Respiratory Biomedical Research Unit, University Hospital Southampton NHS Trust, Southampton, UK. ${ }^{6}$ Gartnavel General Hospital, Glasgow, UK. ${ }^{7}$ Institute of Infection, Immunity and Inflammation, University of Glasgow, Glasgow, UK. ${ }^{8}$ Centre for Biomolecular Sciences, University of Nottingham, Nottingham, UK. 'School of Mathematical Sciences, University of Nottingham, Nottingham, UK.

${ }^{10}$ These authors contributed equally to the study. ${ }^{11} \mathrm{Co}$-senior authors.

Correspondence: Igor L. Chernyavsky, School of Mathematics, University of Manchester, Oxford Road, Manchester M13 9PL, UK. E-mail: Igor.Chernyavskylamanchester.ac.uk

\section{Introduction}

Bronchial thermoplasty (BT) is a non-pharmacological therapy for treating severe asthma by selectively heating conductive airways $(3-10 \mathrm{~mm}$ in diameter) from within the lumen with a low-power electrical current $[1,2]$. During the BT procedure, thermal energy is delivered to the airway wall via a bronchoscope-inserted catheter with a distal basket of four electrodes that expand to make contact with the airway wall, aiming to reach a target temperature of $65^{\circ} \mathrm{C}$ for $10 \mathrm{~s}$.

The primary target of BT is the airway smooth muscle (ASM), a key contributor to airway remodelling, particularly in severe asthma [2-7]. Previous animal studies demonstrated a reduction in airway hyper-responsiveness and an altered ASM histological appearance following BT [8]. Subsequent clinical trials showed improved quality of life and reduced frequency of severe exacerbations in those receiving BT versus a sham procedure, but found no significant difference in lung function as a result of the treatment [9-12]. In uncontrolled observational studies, BT has been associated with an $\sim 50-80 \%$ relative loss of ASM mass determined by bronchial biopsies [13-16], which were typically obtained 1-3 months after completion of the BT procedures. Although thermal ablation by radiofrequency energy is commonly used in surgical practice $[17,18]$, there is a paucity of theoretical [19] and in vitro [20] studies in humans that assess the direct effect of supra-febrile temperatures on ASM cells' survival and function, or the early effects of BT upon ASM mass and epithelial integrity.

We hypothesised that during BT the proportion of the airway exposed to temperatures necessary to affect ASM and epithelial cell survival, determined from in vitro experiments, is sufficient to explain the impact of BT. To test our hypothesis we employed in vitro, in silico and in vivo methodologies to define the acute impact of BT on ASM and epithelial cells.

\section{Methods}

Detailed methods are included in the supplementary material.

In vitro heating of human primary ASM, epithelial cells and bronchial epithelial cells

Primary ASM and epithelial cells were cultured as described previously [21]. The study was approved by the Leicestershire Research Ethics Committee (REC 08/H0406/189). Informed consent was obtained from all subjects. The human bronchial epithelial cells (hBECs) were obtained from LGC Standards (Middlesex, UK).

Cells were grown to confluence in 6- or 24-well plates, then exposed to heated media for $10 \mathrm{~s}$ using the protocol described in the supplementary methods. Heat loss over the 10-s period was measured and showed that following the addition of media heated to $65^{\circ} \mathrm{C}$, cells were exposed to a mean temperature of $58-59^{\circ} \mathrm{C}$ in both 6 - and 24 -well plates (supplementary table S4).

The number of remaining adherent viable cells up to 2 weeks post-heating was assessed using PrestoBlue (Thermo Fisher Scientific, Warrington, UK) according to manufacturer's instructions and confirmed at specified times by cell counts. The percentage of the remaining adherent cell population undergoing apoptosis or necrosis $24 \mathrm{~h}$ post-heating was determined using the Alexa Fluor ${ }^{\oplus} 488$ Annexin V/Dead Cell Apoptosis Kit (Thermo Fisher Scientific) as described previously [22]. Fluorescence emission was collected at $530 \mathrm{~nm}$ (Annexin V) and $>575 \mathrm{~nm}$ (propidium iodide) on a flow cytometer and the percentage of apoptotic and necrotic cells derived respectively using WEASEL ${ }^{\mathrm{TM}}$ software (Frank Battye Flow Cytometry Consulting, Melbourne, Australia).

\section{In silico bioheat mathematical modelling}

Because it is not currently possible to measure the heat transfer within the airway wall during BT in vivo, a two-dimensional mathematical model was developed that couples Joule heating due to the electrical current generated by the BT electrodes (with integrated temperature control feedback, similar to [19]) with 
the bioheat transfer in the airway wall and surrounding parenchymal tissue. The material properties of the lung were applied to the model as shown in supplementary table S1. The coupled model was implemented using the finite element-based modelling framework of COMSOL Multiphysics ${ }^{\circledR} 5.2$ (Stockholm, Sweden). Heat maps were integrated over the airway wall to characterise heating pattern heterogeneity.

Local sensitivity analysis was conducted to identify key geometric, physiological and equipment parameters, and to test the robustness of model predictions. Mathematical model formulation and further technical details can be found in the supplementary material. The mathematical model was validated using the in vitro data on the cooling of heated media in multi-well plates (see the in vitro methods above), which showed good agreement (supplementary figure S3).

The predicted distribution of temperatures in the airway wall, in combination with the thermal dose-dependent response of ASM and bronchial epithelial cells in vitro, was used to estimate the overall acute impact of BT on the bronchial wall (the modelling framework is illustrated in supplementary figure S1).

In vivo response to $B T$

Bronchial biopsies were obtained from 14 subjects before and after BT. All subjects had severe asthma as defined by American Thoracic Society/European Respiratory Society guidelines, and underwent BT as part of their clinical care.

BT was performed as per manufacturer's guidelines over three staged treatment sessions in the following order: right lower lobe, left lower lobe, and both right and left upper lobes. The right middle lobe was not treated owing to the risk of airway collapse and "right middle lobe syndrome" [23]. Biopsies were obtained from the untreated right upper lobe at the first BT session and the treated right lower lobe segmental and subsegmental airways at the second BT session.

Biopsies were embedded in paraffin and $4-\mu \mathrm{m}$ sections stained with haematoxylin and eosin or anti- $\alpha$-smooth muscle actin ( $\alpha$-SMA, clone 1A4; Dako, Stockport, UK). Biopsies were assessed by a single observer (RJR) blinded to clinical characteristics to determine 1) the ASM content as a percentage of total biopsy area; 2) the epithelial integrity by measuring and expressing the length of intact, damaged and denuded epithelium as a percentage of reticular basement membrane length; and 3) the number of myofibroblasts (isolated $\alpha$-SMA-positive stained cells in the lamina propria that were neither located as part of the ASM bundle nor as vascular smooth muscle cells adjacent to vessels) per $\mathrm{mm}^{2}$ of lamina propria.

\section{Statistical analysis}

The statistical analysis is discussed in more detail in the supplementary methods. Briefly, data were analysed in GraphPad Prism ${ }^{\circ} 7.0$ and R project 3.2.4, using parametric and non-parametric tests as appropriate. Confidence intervals for the medians of cell counts were estimated using the bootstrap percentile method ( $\mathrm{R}$ boot package). A p-value $<0.05$ was considered statistically significant.

\section{Results}

In vitro apoptosis and necrosis of ASM and hBEC cells

A metabolic assay demonstrated that the addition of media heated to $65^{\circ} \mathrm{C}$ or $70^{\circ} \mathrm{C}$ for $10 \mathrm{~s}$, but not $45-60^{\circ}$ $\mathrm{C}$, resulted in a significant reduction in the number of ASM and hBEC cells remaining adherent after $24 \mathrm{~h}$ compared to $37^{\circ} \mathrm{C}$ (figure 1a, b). This reduction in the number of viable (metabolically active) adherent cells persisted over 2 weeks after the addition of media heated to $65^{\circ} \mathrm{C}$ or $70^{\circ} \mathrm{C}$ for ASM and to $70^{\circ} \mathrm{C}$ for hBEC cells compared to $37^{\circ} \mathrm{C}$. The number of viable hBEC cells recovered, such that 10 days after the addition of media heated to $65^{\circ} \mathrm{C}$ their numbers were not significantly reduced versus $37^{\circ} \mathrm{C}$ (figure $1 \mathrm{a}-\mathrm{d}$ ). The results presented in figure 1a-d were confirmed at various times using cell counts (figure $1 \mathrm{e}, \mathrm{f}$ ), which supported the results of the metabolic assay. There was a significant reduction in ASM and hBEC cell counts after the addition of media heated to $65^{\circ} \mathrm{C}$ at $24-48 \mathrm{~h}$ (data not shown), which persisted for 1 week (figure 1e) and 2 weeks (data not shown) for ASM, with a recovery of hBEC cell number after 1 week (figure $1 \mathrm{f}$ ). In addition to the reduction in ASM and hBEC cell number post-addition of media heated to $65^{\circ} \mathrm{C}$ or $70^{\circ} \mathrm{C}$ versus $37^{\circ} \mathrm{C}$, there was a significant increase in the percentage of cells undergoing necrosis, but not apoptosis (figure $1 \mathrm{~g}, \mathrm{~h}$ ) in the remaining adherent cell population after $24 \mathrm{~h}$. The median of the relative reduction in viable ASM cell number after the addition of media heated to $65^{\circ} \mathrm{C}$ at $24 \mathrm{~h}$ was $60 \%(95 \%$ bootstrap CI $40-80 \%)$.

\section{In silico heating heterogeneity profiles}

Our computational finite element-based model implementing the BT protocol showed a high degree of temperature variation over an airway wall. An example with reference model geometry (inner and outer wall radii of 2.2 and $3.3 \mathrm{~mm}$, respectively) is shown in figure 2. To assess the impact each of the parameters in the mathematical model had on heat distribution, we undertook a local sensitivity analysis (supplementary material). This demonstrated that the model predictions were relatively insensitive to the 
a)
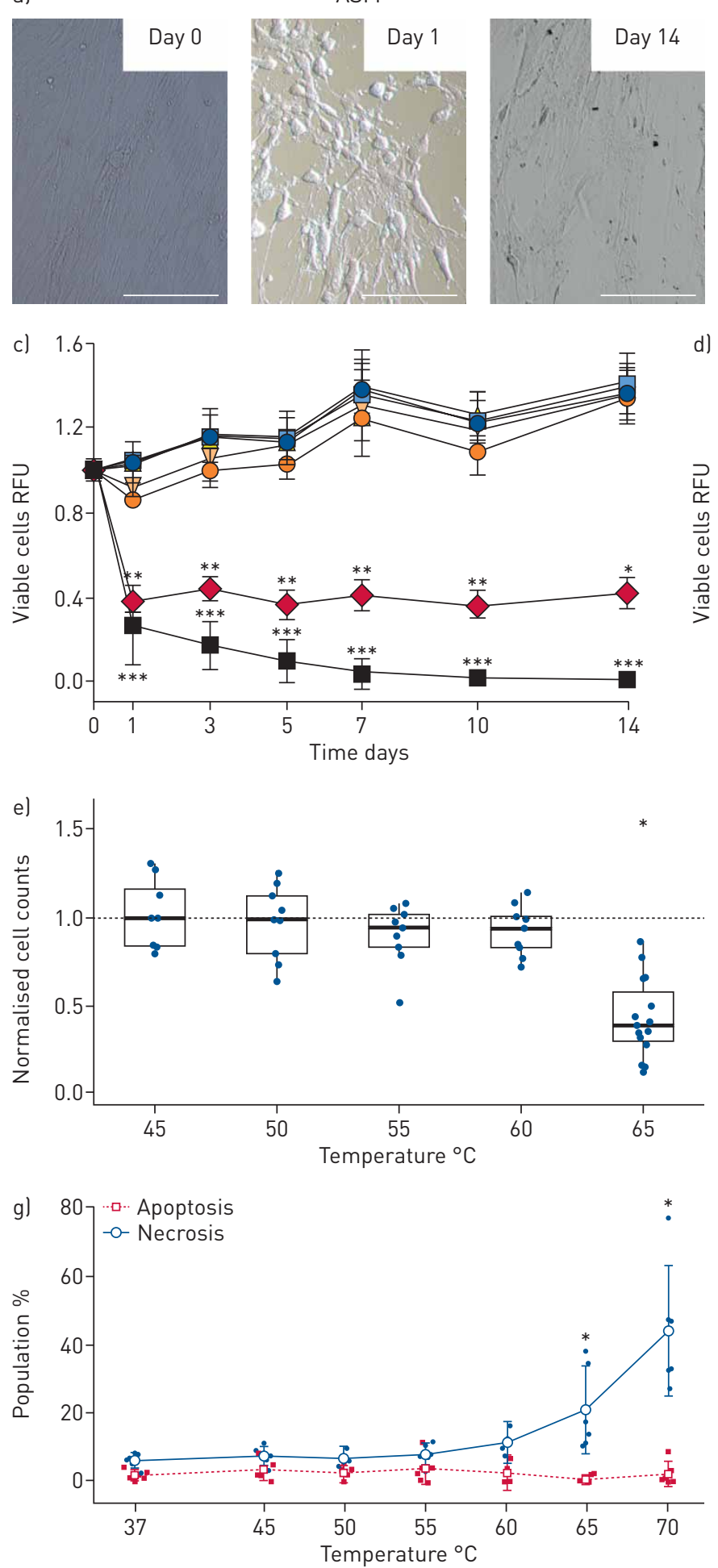

b)

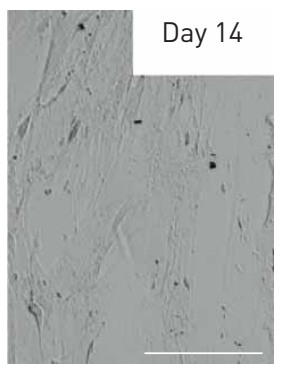

hBEC

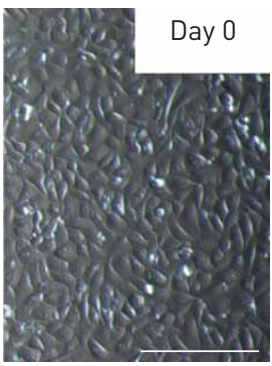

Day 14
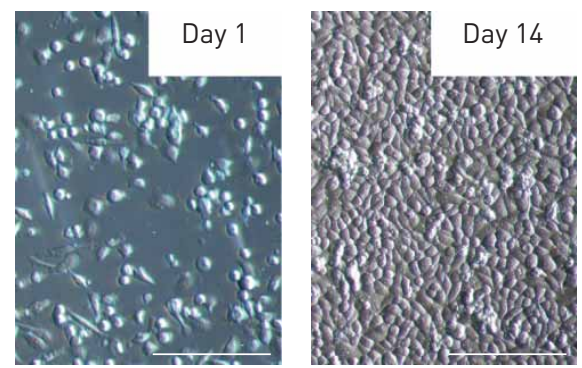
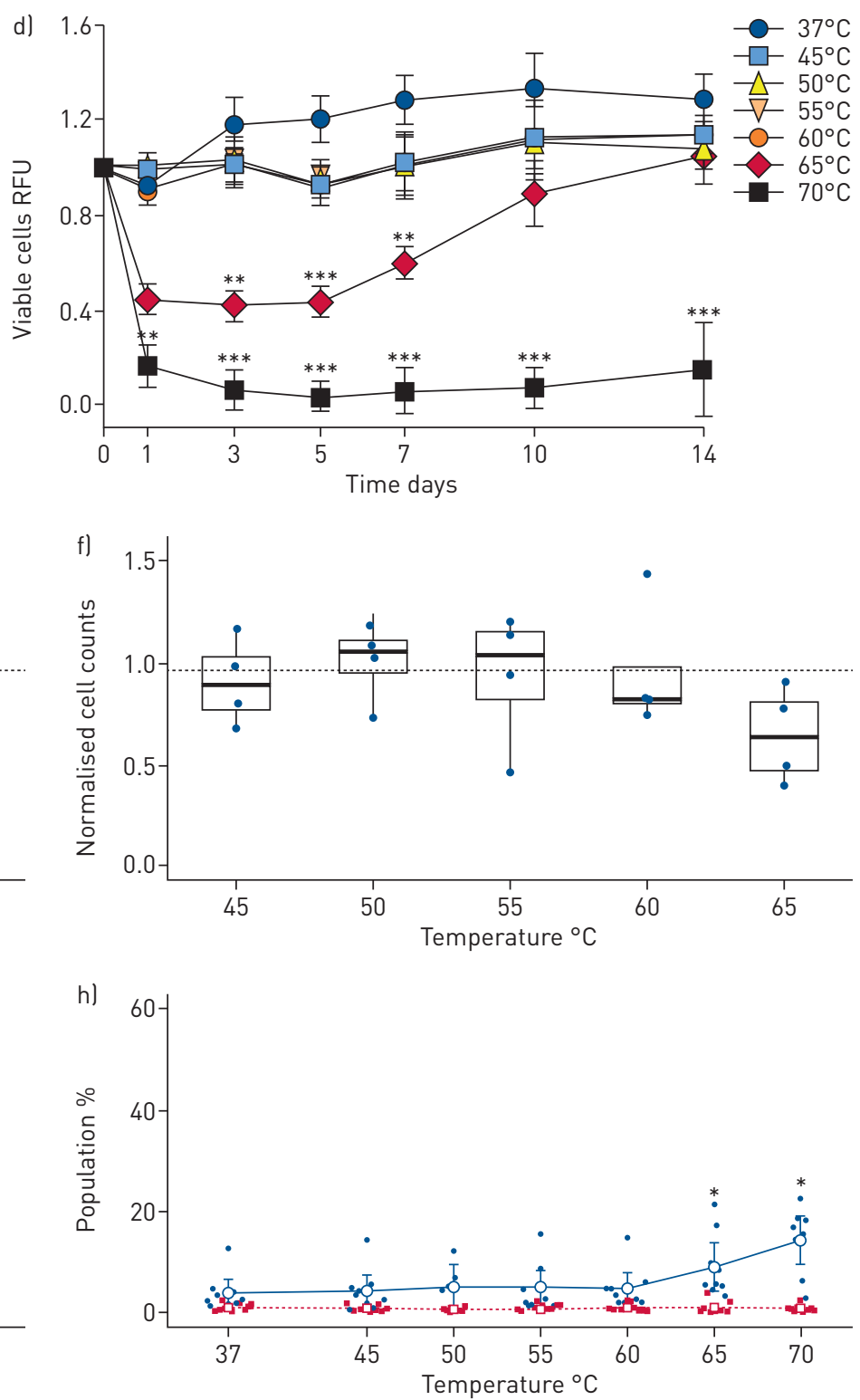

FIGURE 1 Response of in vitro heated airway smooth muscle (ASM) and human bronchial epithelial (hBEC) cells. a, b) Representative cell morphology for cultures following addition of media heated to $65^{\circ} \mathrm{C}$; note the incomplete recovery of ASM (a) compared to hBEC (b) cells over 2 weeks. Scale bar, $0.1 \mathrm{~mm}$. c, d) Longitudinal viability of ASM (c) and hBEC (d) cells following addition of media heated to specified temperatures (mean \pm SE). e, f) Total cell count relative to $37^{\circ} \mathrm{C}$-matched control 1 week after the addition of heated media for ASM (e) and hBEC ( $f$ ) cells. $g$, h) Proportion of apoptotic and necrotic ASM (g) and hBEC (h) cells determined by flow cytometry $24 \mathrm{~h}$ after the addition of media heated to specified temperatures (mean, $95 \% \mathrm{CI}$ ). ${ }^{*} \mathrm{p}<0.05,{ }^{* *} \mathrm{p}<0.01,{ }^{* * *} \mathrm{p}<0.001$ versus $37^{\circ} \mathrm{C}$ controls. 
a)

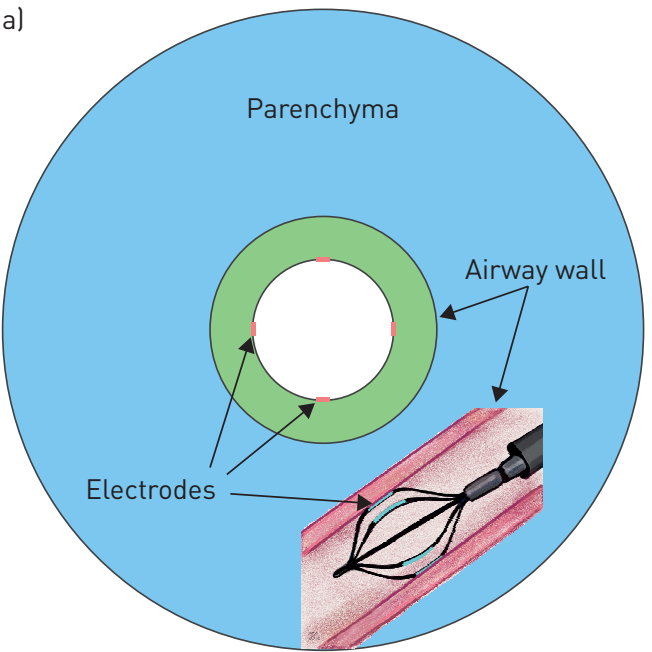

c)

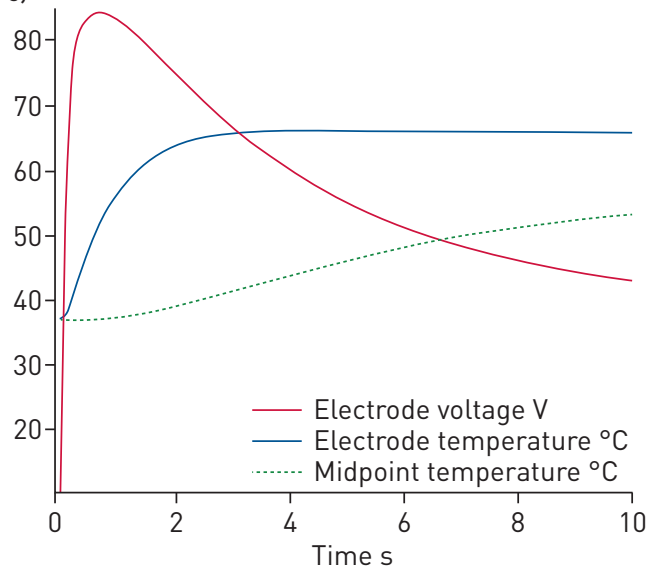

b)

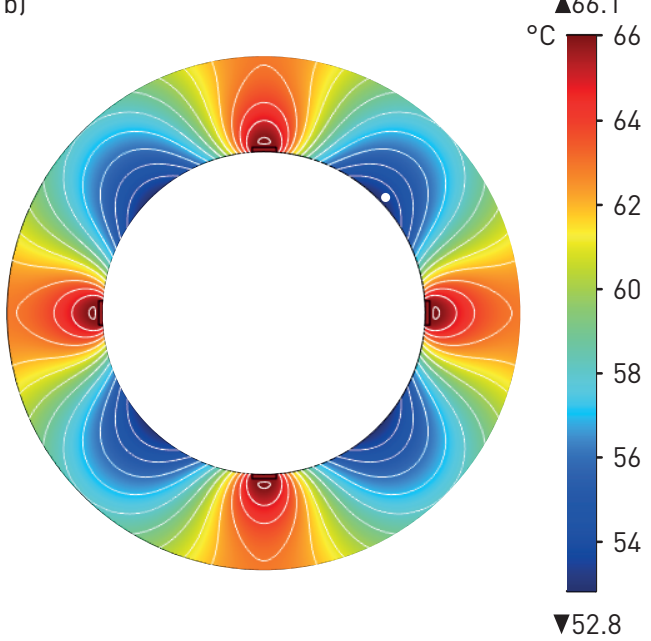

d)

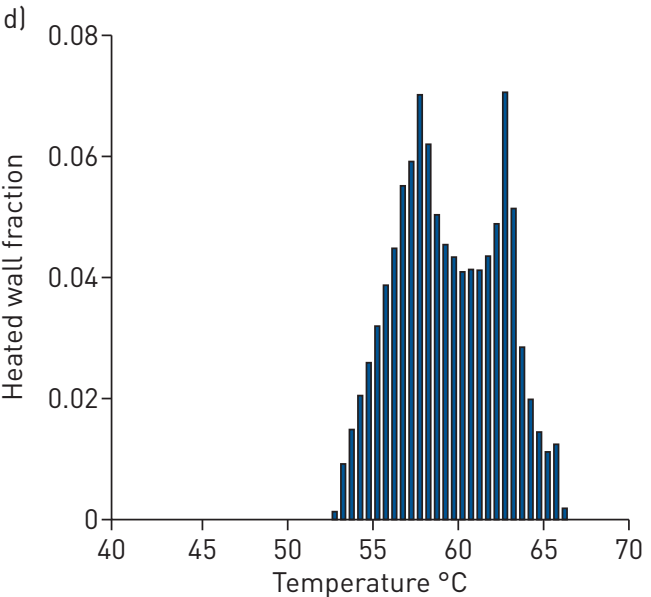

FIGURE 2 Characterisation of bronchial thermoplasty (BT) heating patterns. a) Reference model geometry (inner wall radius of $2.2 \mathrm{~mm}$, outer radius of $3.3 \mathrm{~mm}$ ). b) Heat map at the end of a single BT activation $(10 \mathrm{~s}$ ). c) Temporal dynamics of the applied voltage (red), electrode temperature (solid blue) and temperature at the midpoint between two electrodes (dashed green; marked by a white dot in b). d) Distribution of heated wall area fractions, corresponding to $b$.

tissue material properties and heating control parameters (supplementary table S3), but strongly dependent on the airway calibre and wall thickness (supplementary table S3 and figure S2). This amplification of temperature variation in the larger airways is shown in figure 3 , demonstrating only a small fraction of the wall heated to $65^{\circ} \mathrm{C}$ (figure 3 and table 1).

Although energy transfer is more efficient in the smallest airways accessible to a BT catheter (figure 3a), suboptimal heating is possible in the case of an occluded airway with reduced luminal cooling (figure $3 \mathrm{~b}$ ), and heating heterogeneity is strongly exacerbated in larger airways (figure 3c). Our model suggests that $<5 \%$ of a typical airway (internal radius $\sim 4 \mathrm{~mm}$ ) treated with $\mathrm{BT}$ is exposed to temperatures $>65^{\circ} \mathrm{C}$, and $<10 \%$ to temperatures $>60^{\circ} \mathrm{C}$ (table 1 ). Post-BT thermal equilibration does not improve the extent of heating in the upper temperature range, with no portion of the wall experiencing temperatures $>65^{\circ} \mathrm{C}$ in the 1-2 s after the end of energy delivery (figure $3 \mathrm{~d}, \mathrm{e}$ ), even when there is no volumetric cooling due to tissue perfusion and alveolar moisture evaporation. Therefore, only a small percentage of the area of airways treated with BT are likely to be exposed to temperatures $>60^{\circ} \mathrm{C}$, except for the smallest treated airways. Owing to the accessibility of the airways with a bronchoscope, those airways biopsied were proximal to the smallest airways treated with BT.

In vivo BT impact on ASM mass and epithelial integrity

The baseline and follow-up clinical characteristics of the 14 subjects are shown in table 2. Nine subjects were receiving treatment with regular systemic corticosteroids (Global Initiative for Asthma (GINA) step 5) and the remaining subjects were receiving GINA step 4 treatment. Six weeks after the last BT 

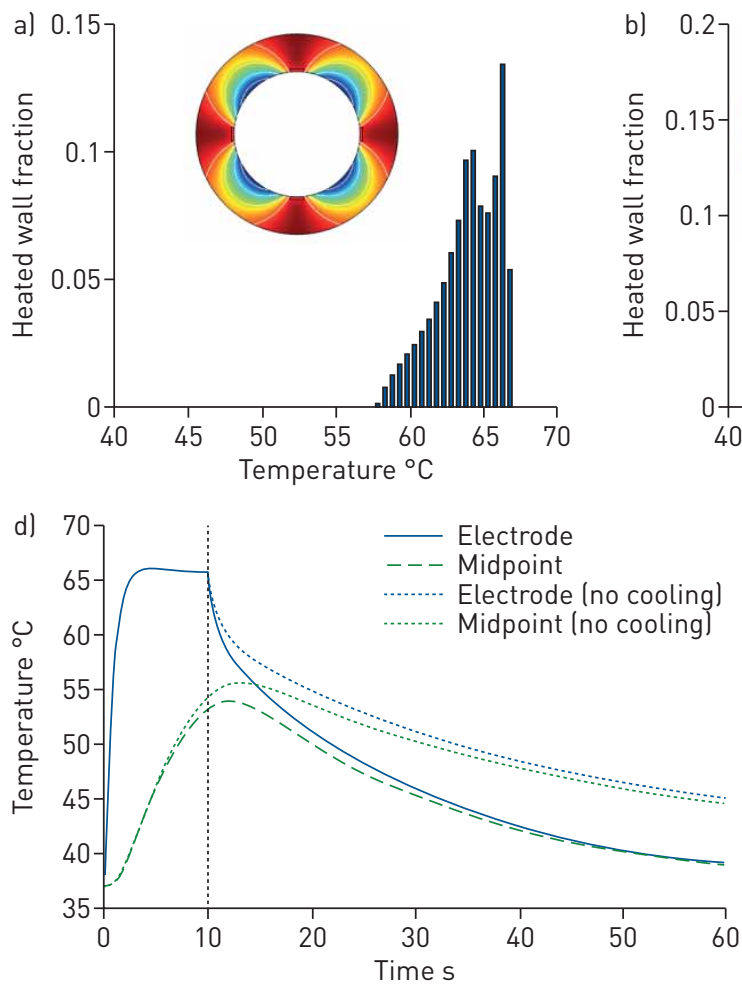
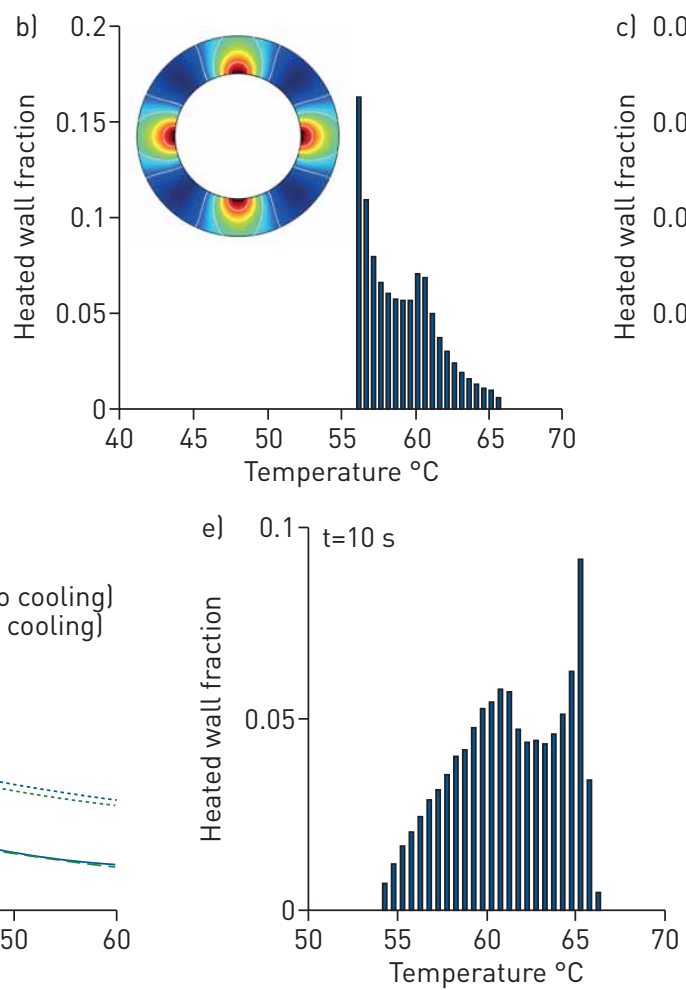
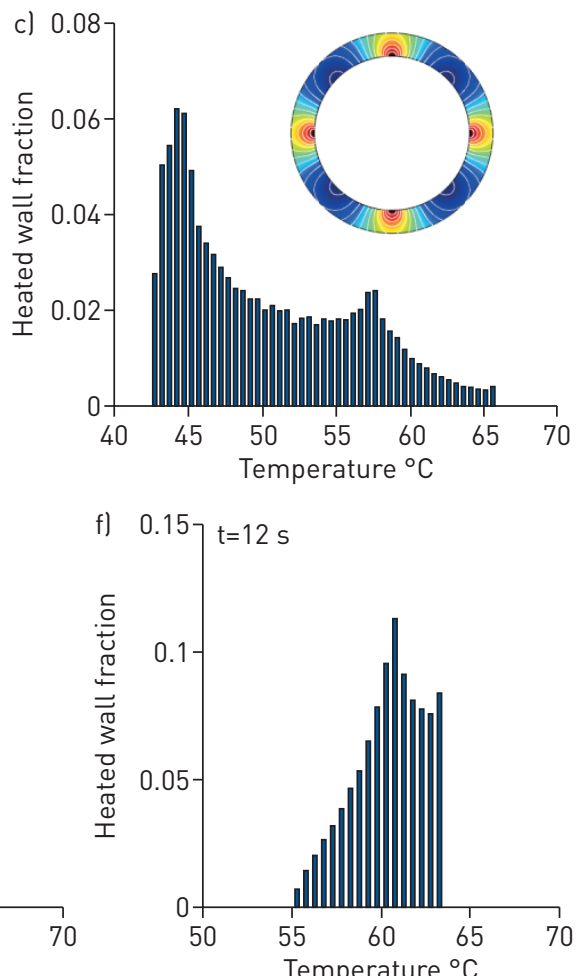

FIGURE 3 Airway temperature heterogeneity across bronchial generations and heating scenarios. Heating patterns a) at the lowest end of bronchial thermoplasty (BT) applicability (luminal radius of $1.5 \mathrm{~mm}$ ); b) for a midrange airway (luminal radius of $2.2 \mathrm{~mm}$, corresponding to figure $2 \mathrm{~b}, \mathrm{~d}$ ) with impeded luminal evaporative cooling (e.g. occluded with a bronchoscope); and c) for a larger airway (luminal radius of $4.4 \mathrm{~mm}$ ). d) Thermal dynamics of an airway wall after the end of a single BT activation (marked by vertical dashed line) for the reference case of figure 2 (solid and dashed) and for the case of absent tissue perfusion and evaporative cooling (dotted lines). e, f) Temperature distributions at $10 \mathrm{~s}$ (e) and $12 \mathrm{~s}(\mathrm{f})$, corresponding to the case of absent volumetric tissue cooling.

intervention there was no change in lung function, whereas scores for both the Asthma Control Questionnaire-6 (ACQ6) and Asthma Quality of Life Questionnaire (AQLQ) significantly improved by more than the clinically important difference of 0.5 (ACQ6 mean difference $-0.7,95 \%$ CI -1.3 to -0.1 , $\mathrm{p}=0.03$; AQLQ mean difference $0.8,95 \%$ CI 0.1 to $1.5, \mathrm{p}=0.03$ ) (table 2).

The median time between baseline and follow-up biopsies was 28 days (range 14-56 days). A representative bronchial biopsy is shown in figure $4 \mathrm{a}$. There was a reduction in median ASM mass from $12 \%$ (interquartile range (IQR) 6-17) pre-BT to 6\% (IQR 1-10) post-BT (median difference 5\%, IQR $0-10$, Wilcoxon $\mathrm{p}=0.03$; figure $4 \mathrm{~b}$ ). The median relative reduction in ASM mass was 58\% (IQR 6-90).

There was also a significant improvement in median epithelial integrity from $29 \%$ (IQR $15-40$ ) pre-BT to $46 \%$ (IQR 25-56) post-BT (median improvement 14\%, IQR 6-29, p=0.007; figure 4c, d). The median relative increase in epithelial integrity was 56\% (IQR 19-120).

TABLE 1 Quantification of simulated thermal impact of bronchial thermoplasty at the end of an activation cycle

$\frac{\text { Intermediate conducting airway }{ }^{\#}}{\text { Baseline } \begin{array}{c}\text { Occluded (no evaporative } \\ \text { cooling) }\end{array}}$

Large conducting airway ${ }^{\text {I }}$ cooling)

$\begin{array}{lccc}\text { Mean wall temperature }{ }^{\circ} \mathbf{C} & 59 & 59 & 50 \\ \text { Wall area fraction heated }>\mathbf{6 5} 5^{\circ} \mathbf{C} \% & 3 & 2 & 1 \\ \text { Wall area fraction heated }>\mathbf{6 0} 0^{\circ} \mathbf{C} \% & 43 & 35 & 7 \\ \text { Wall area fraction heated }>\mathbf{5 5} 5^{\circ} \mathbf{C} \% & 93 & 100 & 25\end{array}$

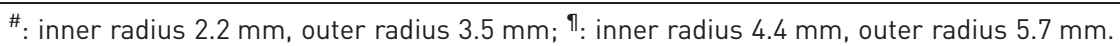


TABLE 2 Baseline and follow-up (median time of 28 days) clinical characteristics of patients undergoing thermoplasty and biopsy

\begin{tabular}{|c|c|c|c|}
\hline Characteristic & Baseline & Follow-up & p-value \\
\hline Subjects $\mathrm{n}$ & 14 & - & \\
\hline Female sex $n$ & 9 & - & \\
\hline Age years & $52 \pm 13$ & - & \\
\hline GINA classification $5 \mathrm{n}$ & 9 & - & \\
\hline BMI $\mathrm{kg} \cdot \mathrm{m}^{-2}$ & $31 \pm 8$ & - & \\
\hline Exacerbations in last 12 months $n$ & $4 \pm 3$ & - & \\
\hline Pre-BD FEV $1 \%$ predicted & $68 \pm 19$ & $67 \pm 20$ & 0.5 \\
\hline Post-BD FEV1/FVC \% & $63 \pm 12$ & $63 \pm 12$ & 0.8 \\
\hline Bronchodilator reversibility \% & $19 \pm 11$ & $16 \pm 12$ & 0.5 \\
\hline ACQ6 score & $3.1 \pm 1.6$ & $2.5 \pm 1.7^{*}$ & 0.03 \\
\hline AQLQ score & $3.4 \pm 1.7$ & $4.1 \pm 1.8^{*}$ & 0.03 \\
\hline
\end{tabular}

Data are presented as mean $\pm S D$, unless otherwise indicated. GINA: Global Initiative for Asthma; BMI: body mass index; BD: bronchodilator; FEV1: forced expiratory volume in $1 \mathrm{~s}$; FVC: forced vital capacity; ACQ6: Asthma Control Questionnaire-6; AQLQ: Asthma Quality of Life Questionnaire. *: paired $t$-test $p<0.05$ compared to baseline.

There was a numerical but nonsignificant reduction in the number of sub-epithelial myofibroblasts ( pre-BT median 25 cells $\cdot \mathrm{mm}^{-2}$, IQR $7-47$ versus post-BT median 13 cells $\cdot \mathrm{mm}^{-2}$, IQR $6-21 ; \mathrm{p}=0.17$ ). There was a significant inverse correlation between the change in ASM mass and myofibroblast count in the lamina propria following BT (Spearman $r=-0.55, \mathrm{p}=0.046$; figure $4 \mathrm{e}$ ). Change in epithelial integrity did not correlate with change in myofibroblast count nor ASM mass following BT (data not shown).

Improvements in AQLQ score did not correlate with pre-BT epithelial integrity, ASM mass or myofibroblast number in the lamina propria nor with post-BT change (data not shown). Only three subjects had an improvement $\geqslant 1.0$ thus no responder analysis was undertaken. Pre-BT epithelial integrity, ASM mass and myofibroblast number in the lamina propria were not related to change in ACQ6 score (data not shown). The change in ACQ6 score was inversely related to the change in ASM mass (Spearman $r=-0.67, \mathrm{p}=0.018$ ), but not with epithelial integrity $(r=-0.03, \mathrm{p}=0.09)$ or myofibroblast number in the lamina propria $(r=0.41$, $\mathrm{p}=0.18$ ). Five subjects had an improvement in ACQ6 score $\geqslant 1.0$. Compared with subjects not showing this improvement in ACQ6, these five subjects had a small increase in ASM mass post-BT (median 2\%, IQR -2 to 8 versus median $-10 \%$, IQR -8 to $-12 ; \mathrm{p}=0.003$ ). This was in contrast to a higher pre-BT myofibroblast number in the lamina propria (59 cells $\cdot \mathrm{mm}^{-2}$, IQR $36-84$ versus 7 cells $\cdot \mathrm{mm}^{-2}$, IQR $3-43$; $\mathrm{p}=0.03$ ) and greater decrease post-BT ( 37 cells. $\mathrm{mm}^{-2}$, IQR $30-51$ versus -8 cells $\cdot \mathrm{mm}^{-2}$, IQR 3 to $-27 ; \mathrm{p}=0.048$ ). Those with both an increase in ASM mass and a decrease in myofibroblast number in the lamina propria had the greatest improvement in ACQ6 score compared with those that either had a decrease in both or a decrease in ASM mass and increase in myofibroblast number (figure 4e).

\section{Discussion}

We have developed an integrated in vitro and in silico framework to model the acute effects of BT on ASM and epithelial cells. In this framework, the in silico mathematical model serves as a "bridge" between the in vitro and in vivo thermal effects, which are inaccessible by other means. The in vitro model identified a sharp threshold in the response of both hBEC and ASM cells to heating. In vitro hBEC and ASM cell number decreased significantly after the addition of media heated to $\geqslant 65^{\circ} \mathrm{C}$. Importantly, taking into account the heat loss over $10 \mathrm{~s}$ in the in vitro experiments, these cells were exposed to a mean temperature of $58-59^{\circ} \mathrm{C}$. The mathematical model predicted a localised and highly heterogeneous heating pattern that is very sensitive to airway calibre, with a relatively small fraction of the bronchial wall heated to $>60^{\circ} \mathrm{C}$ in all but the smallest airways (see also [25]). The integrated in vitro and in silico model predictions were tested against in vivo bronchial biopsies taken pre- and post-BT. The biopsy samples showed an increase in epithelial integrity and a reduction in ASM mass after BT.

Although greater than predicted based on our mathematical model, the observed post-BT relative median reduction in ASM mass of 58\% was consistent with previous clinical studies [13-16]. To explain this level of ASM reduction by acute thermal injury alone, most of the airway wall in the calibre of airways sampled at bronchoscopy would need to be heated to $\geqslant 60^{\circ} \mathrm{C}$. Thus, if the in vitro and in silico predictions are correct, the difference must be due to an alternative biological mechanism triggered in response to BT, such as the active thermal bystander effect [26]. 

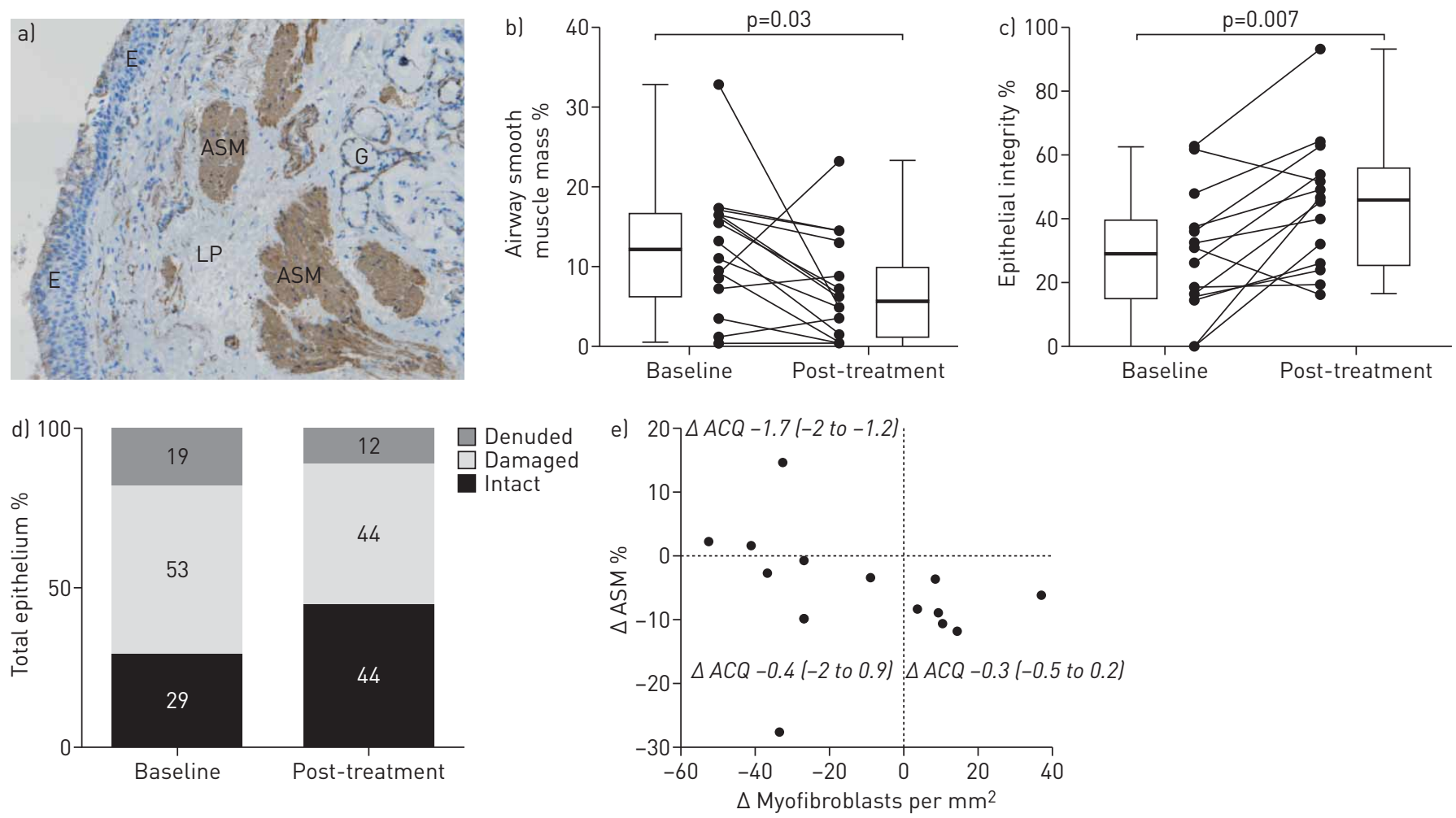

FIGURE 4 Histology analysis of airway smooth muscle (ASM) content and epithelial integrity in bronchial biopsies (at baseline and at about 1 month post-bronchial thermoplasty (BT)). a) Example endobronchial biopsy stained for $\alpha$-smooth muscle actin (E: epithelium; LP: lamina propria; G: gland). b) ASM mass \% pre- and post-BT ( $p<0.05)$. c) Epithelial integrity pre- and post-BT ( $<<0.01)$. In b and $c$, the horizontal line represents the median, the box represents the interquartile range (IQR) and the whiskers represent the minimum and maximum. d) Detailed breakdown of epithelial structure at baseline and post-BT (mean). The total percentage for the baseline does not equal $100 \%$ owing to rounding. e) Change in ASM mass versus the number of myofibroblasts per $\mathrm{mm}^{2}$ of lamina propria following BT (Spearman's rank correlation $r=-0.55$, $\mathrm{p}=0.046$ ), with change in Asthma Control Questionnaire-6 (ACQ6) score (mean (IQR)) reported for each response subgroup (quadrants).

Our in vivo biopsies showed significantly improved epithelial integrity after BT, which likely reflects epithelial repair in response to thermal injury. Indeed, our in vitro data show evidence of acute phase epithelial repair in response to heat. Others studies have not found changes in the epithelial phenotype in biopsies obtained 3 months after completion of BT treatment, but have reported other effects upon collagen deposition and bronchial nerves [16]. Although in this small study a reduction in ASM mass did not correlate with increased epithelial integrity, whether epithelial repair might impact on other changes in airway wall structure, including ASM mass, following BT warrants further investigation.

Myofibroblast numbers are increased in the lamina propria in patients with asthma and they traffic to sites of injury, differentiate and promote wound repair [27-29]. We considered whether BT might affect the number of myofibroblast cells in the lamina propria, and whether the prevalence of these cells might relate to changes observed in the epithelium and ASM. We identified a numerical but nonsignificant reduction in the overall number of myofibroblasts in the lamina propria in response to BT. However, there was a significant inverse correlation between the change in ASM mass and myofibroblast number after BT. This might represent a dynamic relationship between myofibroblasts and the ASM bundle, with migration of myofibroblasts to and from the ASM bundle. Despite the small number of subjects in our study, we were able to explore the relationship between the effects of BT on ASM mass, epithelial integrity and myofibroblast numbers in the lamina propria and asthma-related symptoms assessed 6 weeks after the last BT intervention. Surprisingly we found that improvement in asthma control was inversely related to post-BT change in ASM mass, and that improvement in asthma control was greatest in those with an increase in ASM mass and a reduction in myofibroblast number. The observed epithelial repair was not associated with improved asthma control, but whether epithelial repair contributes to the reduced exacerbation rates observed in larger studies after BT needs to be further investigated.

Our study had a number of potential limitations. The in vitro studies could not fully recapitulate the behaviour of the ASM cells and bronchial epithelial cells in vivo because the heated media was added directly to specific cell types in isolation. They also did not take into account asthmatic versus 
non-asthmatic cellular phenotype, cell-cell interactions or the presence of submucosal tissue. In addition, there was a small amount of heat loss over the 10-s exposure in the model system. However, our in vitro data were very consistent across different methodological approaches and between two centres.

The mathematical model involved a number of simplifying assumptions. We modelled an airway and surrounding parenchyma as a cross-section and neglected three-dimensional effects. This approximation is justified by the relatively long $(\sim 5 \mathrm{~mm})$ length of the electrode compared to the airway wall thickness $(\sim 1 \mathrm{~mm})$. The computational model also used averaged homogeneous electrothermal material properties of the airway wall and parenchymal tissues and did not account for possible anatomo-physiological variations within and between treated individuals. There was also the possibility of inherent operator variability, patient lung movement and complex automatic controller safeguards incorporated into the BT protocol, which were not included in the model. We have, however, tested the robustness of the in silico model and quantified the uncertainty associated with model predictions via 1) appropriate mesh convergence tests, 2) application of random spatial perturbations in tissue material properties (results not shown) and 3) a comprehensive parameter sensitivity analysis. Indeed, the sensitivity analyses showed that model predictions remained unaffected by a moderate level of variability in tissue material properties, whereas airway wall and luminal morphometry had the greatest impact on the model. Nonetheless, the developed mathematical modelling framework is intended to provide a qualitative rather than quantitative insight into the impact of BT. Thus, notwithstanding the limitations of the in vitro and in silico modelling, we are confident that these data do not support the concept of substantial acute loss of ASM mass as a direct response to the heating effect of BT in the airways sampled at bronchoscopy.

There were also shortcomings in the BT in vivo clinical trial. First, the in vivo bronchial biopsies were taken from the right upper lobe at baseline and the right lower lobe following BT, and the ASM mass at baseline was lower than we have previously reported [24]. It is possible that some of the changes demonstrated were due to variability in baseline remodelling between lung lobes, and variability in subject selection in this cohort compared to others. We also observed a high degree of inter-patient variability in the ASM response to BT. However, despite these factors, the observed overall magnitude of ASM mass reduction was similar to that given in previous reports, giving confidence to the likelihood of the observed changes being genuine. Finally, our BT in vivo study was too small to determine whether the observed changes in airway remodelling relate to future clinical risk such as exacerbations. Determining this requires either a large prospective study or a meta-analysis of the reported BT biopsy studies.

In conclusion, our in vivo data support a reduction in ASM mass in bronchial biopsies obtained post-BT but our combined in vitro and in silico modelling suggest that the extent of this reduction in ASM mass cannot be entirely explained by a direct acute effect of thermal injury on ASM following BT. Although we cannot exclude the possibility that peri-procedural prednisolone contributed to remodelling, prednisolone was administered prior to all procedures and, importantly, its effects on the epithelium are inconsistent and no effects on the ASM mass have previously been reported [30]. Our data therefore challenge the current concepts of the potential mechanisms of BT, indicating that an alternative mechanism(s) besides direct thermal injury may contribute to this process. Epithelial integrity was also shown to increase in response to $\mathrm{BT}$, and post-BT myofibroblast number in the lamina propria was inversely related to ASM mass. Whether epithelial repair in response to thermal injury and/or the dynamic interaction between the ASM and myofibroblasts have consequent effects upon BT-associated reduced ASM mass remains to be confirmed. Whether the BT protocol can be optimised to target specific airways or elements of airway remodelling, perhaps in combination with patient-specific modelling to facilitate precision medicine, needs to be studied.

Acknowledgements: The authors would like to thank Oliver Jensen, Ian Jones and Timothy Waite for their advice and critical review of the manuscript; all AirPROM Consortium members for stimulating discussions at various stages of the study; and Davinder Kaur and Michael Biddle for assistance with the in vitro experiments.

Author contributions: I.L. Chernyavsky and B.S. Brook took part in mathematical model design; I.L. Chernyavsky performed numerical simulations; I.L. Chernyavsky, R.M. Saunders, G.E. Morris, F.R.A.J. Rose, S. Siddiqui, B.S. Brook and C.E. Brightling conceived the experimental model; R.M. Saunders and G.E. Morris conducted in vitro experiments; I.L. Chernyavsky, R.J. Russell and R.M. Saunders performed statistical data analysis; R. Berair, A. Singapuri, A.H. Mansur, P.H. Howarth, P. Dennison, R. Chaudhuri, S. Bicknell, S. Siddiqui and C.E. Brightling coordinated the clinical trial and undertook procedures; R.J. Russell, R. Berair and L. Chachi analysed patient biopsy data; I.L. Chernyavsky, R.J. Russell, R.M. Saunders, S. Siddiqui, B.S. Brook and C.E. Brightling interpreted the results and prepared the manuscript. All authors read and approved the final manuscript.

Support statement: The work was part supported by AirPROM 7th EU Framework grant 270194 (all authors), Medical Research Council (MRC) grant MR/N011538/1 (I.L. Chernyavsky), MRC grant MR/M004643/1 (B.S. Brook), Wellcome Trust Senior Fellowship WT082265 (C.E. Brightling) and by National Institute for Health (NIHR) Leicester Biomedical 
Research Centre. The views expressed are those of the authors and not necessarily those of the NHS, the NIHR or the Department of Health. Funding information for this article has been deposited with the Crossref Funder Registry.

Conflict of interest: R.M. Saunders reports grants from 7th EU Framework, Wellcome Trust and National Institute for Health Research, during the conduct of the study. A.H. Mansur has received an educational grant for service support from AstraZeneca Pharmaceuticals, and received fees for talks and advisory group contribution and conference attendance from Novartis, GlaxoSmithKline, AstraZeneca, Napp Pharmaceuticals, Boehringer Ingelheim and others, outside the submitted work. P.H. Howarth reports grants from the European Union (AirPROM collaborative grant), during the conduct of the study. R. Chaudhuri reports being an advisory board member for GlaxoSmithKline, AstraZeneca, Teva Pharmaceutical Industries and Novartis and receiving educational grants for her institute from Novartis; receiving fees for speaking at meetings organised by GlaxoSmithKline, AstraZeneca, Chiesi and for attending international conferences sponsored by Novartis, Teva Pharmaceutical Industries, AstraZeneca and Boehringer Ingelheim. S. Siddiqui reports personal fees for advisory board participation from AstraZeneca and Boehringer Ingelheim, personal fees for advisory/consulting from Owlstone Nanotech and Mundipharma, speaker fees from Novartis, grants for imaging research in asthma from Napp Pharmaceuticals, and speaker fees from the European Respiratory Society, outside the submitted work. C.E. Brightling has received, paid to his institution, grants and consultancy fees from GlaxoSmithKline, Novartis, Chiesi, MedImmune/AstraZeneca, Boehringer Ingelheim, MSD Pharmaceuticals, PrEP Biopharm, Vectura, Teva Pharmaceutical Industries, Sanofi, Regeneron and Roche/Genentech. I.L. Chernyavsky reports research fellowship support from the European Commission (FP7 AirPROM) and a grant from Medical Research Council UK (New Investigator Research Grant), during the conduct of the study.

\section{References}

1 US FDA. Alair Bronchial Thermoplasty System. Summary of safety and effectiveness data. 2010. www.accessdata. fda.gov/cdrh_docs/pdf8/P080032b.pdf Date last accessed: April 24, 2018.

2 Wilhelm CP, Chipps BE. Bronchial thermoplasty: a review of the evidence. Ann Allergy Asthma Immunol 2016; 116: 92-98.

3 Seow CY, Fredberg JJ. Historical perspective on airway smooth muscle: the saga of a frustrated cell. J Appl Physiol 2001; 91: 938-952.

4 Mitzner W. Airway smooth muscle: the appendix of the lung. Am J Respir Crit Care Med 2004; 169: 787-790.

5 Zuyderduyn S, Sukkar MB, Fust A, et al. Treating asthma means treating airway smooth muscle cells. Eur Respir J 2008; 32: 265-274

6 Janssen LJ. Airway smooth muscle as a target in asthma and the beneficial effects of bronchial thermoplasty. J Allergy 2012; 2012: 593784.

7 Laxmanan B, Hogarth DK. Bronchial thermoplasty in asthma: current perspectives. J Asthma Allergy 2015; 8: 39-49.

8 Danek CJ, Lombard CM, Dungworth DL, et al. Reduction in airway hyperresponsiveness to methacholine by the application of RF energy in dogs. J Appl Physiol 2004; 97: 1946-1953.

9 Cox G, Miller JD, McWilliams A, et al. Bronchial thermoplasty for asthma. Am J Respir Crit Care Med 2006; 173 965-969.

10 Pavord ID, Cox G, Thomson NC, et al. Safety and efficacy of bronchial thermoplasty in symptomatic, severe asthma. Am J Respir Crit Care Med 2007; 176: 1185-1191.

11 Castro M, Rubin AS, Laviolette M, et al. Effectiveness and safety of bronchial thermoplasty in the treatment of severe asthma. Am J Respir Crit Care Med 2010; 181: 116-124.

12 Bellanti JA, Settipane RA. Bronchial thermoplasty: quo vadis? Allergy Asthma Proc 2015; 36: 240-240.

13 Pretolani M, Dombret M-C, Thabut G, et al. Reduction of airway smooth muscle mass by bronchial thermoplasty in patients with severe asthma. Am J Respir Crit Care Med 2014; 190: 1452-1454.

14 Chakir J, Haj-Salem I, Gras D, et al. Effects of bronchial thermoplasty on airway smooth muscle and collagen deposition in asthma. Ann Am Thorac Soc 2015; 12: 1612-1618.

15 Denner DR, Doeing DC, Hogarth DK, et al. Airway inflammation after bronchial thermoplasty for severe asthma. Ann Am Thorac Soc 2015; 12: 1302-1309.

16 Pretolani M, Bergqvist A, Thabut G, et al. Effectiveness of bronchial thermoplasty in patients with severe refractory asthma: clinical and histopathologic correlations. J Allergy Clin Immunol 2017; 139: 1176-1185.

17 Berjano EJ Theoretical modeling for radiofrequency ablation: state-of-the-art and challenges for the future. Biomed Eng Online 2006; 5: 24.

18 Slebos D-J, Klooster K, Koegelenberg CFN, et al. Targeted lung denervation for moderate to severe COPD: a pilot study. Thorax 2015; 70: 411-419.

19 Jarrard J, Wizeman B, Brown R, et al. A theoretical model of the application of RF energy to the airway wall and its experimental validation. Biomed Eng Online 2010; 9: 81.

20 Dyrda P, Tazzeo T, DoHarris L, et al. Acute response of airway muscle to extreme temperature includes disruption of actin-myosin interaction. Am J Respir Cell Mol Biol 2011; 44: 213-221.

21 Kaur D, Gomez E, Doe C, et al. IL-33 drives airway hyper-responsiveness through IL-13-mediated mast cell: airway smooth muscle crosstalk. Allergy 2015; 70: 556-567.

22 Hollins F, Kaur D, Yang W, et al. Human airway smooth muscle promotes human lung mast cell survival, proliferation, and constitutive activation: cooperative roles for CADM1, stem cell factor, and IL-6. J Immunol 2008; 181: 2772-2780.

23 Dombret M-C, Alagha K, Boulet LP, et al. Bronchial thermoplasty: a new therapeutic option for the treatment of severe, uncontrolled asthma in adults. Eur Respir Rev 2014; 23: 510-518.

24 Berair R, Hartley R, Mistry V, et al. Associations in asthma between quantitative computed tomography and bronchial biopsy-derived airway remodelling. Eur Respir J 2017; 49: 1601507.

25 Boulet L-P, Laviolette M. Acute effects of bronchial thermoplasty: a matter of concern or an indicator of possible benefit to small airways? Eur Respir J 2017; 49: 1700029.

26 Purschke M, Laubach H-J, Anderson RR, et al. Thermal injury causes DNA damage and lethality in unheated surrounding cells: active thermal bystander effect. J Investig Dermatol 2010; 130: 86-92. 
27 Kaur D, Saunders R, Berger P, et al. Airway smooth muscle and mast cell-derived CC chemokine ligand 19 mediate airway smooth muscle migration in asthma. Am J Respir Crit Care Med 2006; 174: 1179-1188.

28 Hinz B. The role of myofibroblasts in wound healing. Curr Res Transl Med 2016; 64: 171-177.

29 Gerarduzzi C, Di Battista JA. Myofibroblast repair mechanisms post-inflammatory response: a fibrotic perspective. Inflamm Res 2017; 66: 451-465.

30 Berair R, Brightling CE. Asthma therapy and its effect on airway remodelling. Drugs 2014; 74: 1345-1369. 